\title{
Les livres allegati : un nouveau média pour la littérature
}

Sandro Baffi

\section{OpenEdition}

Journals

Édition électronique

URL : http://journals.openedition.org/cei/136

DOI : $10.4000 /$ cei. 136

ISSN : 2260-779X

Éditeur

UGA Éditions/Université Grenoble Alpes

Édition imprimée

Date de publication : 15 juin 2010

Pagination : 183-193

ISBN : 978-2-84310-168-7

ISSN : 1770-9571

\section{Référence électronique}

Sandro Baffi, «Les livres allegati : un nouveau média pour la littérature », Cahiers d'études italiennes [En ligne], 11 | 2010, mis en ligne le 15 décembre 2011, consulté le 27 mars 2021. URL : http:// journals.openedition.org/cei/136 ; DOI : https://doi.org/10.4000/cei.136 


\title{
LES LIVRES ALLEGATI : UN NOUVEAU MÉDIA POUR LA LITTÉRATURE
}

\author{
Sandro Baffi \\ Université Paris 4
}

Le développement des nouvelles technologies permet aujourd'hui à la littérature d'atteindre son public à travers de nouveaux canaux, en particulier ceux des médias électroniques et informatiques. À côté de ces médias nouveaux, au cours des dernières années, la littérature a emprunté un circuit qui n'est pas nouveau, mais qui est une forme nouvelle d'un média ancien : les livres allegati ${ }^{\perp}$. Il s'agit en effet d'un phénomène récent, apparu pour la première fois en Italie de façon sporadique entre 1992 et 1996, de façon généralisée à partir de 2002, qui fait partie d'un ensemble plus vaste de «marketing culturel» $i$ collaterali. Il a une importance économique considérable et des conséquences non négligeables sur le plan culturel. Il s'insère dans la longue histoire des rapports à la fois féconds et ambigus entre la littérature et les canaux par lesquels elle atteint ses lecteurs, en particulier entre la littérature et la presse; par là même, il brouille les pistes et contribue à troubler la distinction traditionnelle entre littérature savante ou légitimée et littérature populaire ou de masse, en somme entre littérature et "paralittératures", pour utiliser une notion contestée mais opératoire.

Notre approche sera essentiellement civilisationnelle. Nous nous intéresserons plus particulièrement à l'aspect éditorial de ce phénomène et, à la lumière d'une initiative récente, au rapport nouveau qui peut s'instaurer entre l'auteur et son ouvre, entre l'auteur et ses lecteurs.

I. Pour désigner ces livres nous garderons l'adjectif italien car le français ne dispose pas (pas encore?) d'une expression équivalente. Les produits (livres, DVD, CD, etc.) vendus avec les journaux, moyennant un supplément de prix, sont appelés «plus-produits» ou "produits dérivés» (collaterali en italien). 


\section{Édition et médias}

Mercredi I6 janvier 2002: La Repubblica lance sa première collection de 50 livres, "Il Novecento in 50 romanzi », en distribuant gratuitement le premier numéro de cette collection : Le nom de la Rose d'Umberto Eco. Le succès est énorme car tous les exemplaires disponibles (I million) disparaissent en une matinée et il faudra réimprimer rapidement le roman; au total I,2 million d'exemplaires seront distribués.

Ce succès ne se démentira pas au cours des semaines suivantes, car tous les jeudis, allegati au quotidien, au prix de 4,90 euros (plus le prix $\mathrm{du}$ journal, 5,80 euros en tout) les 50 titres se vendront en moyenne à 475000 exemplaires pour un total, sur l'année et pour toute la collection, de 25 millions de livres!

Quatre mois plus tard, Il Corriere della Sera, le quotidien antagoniste de La Repubblica, démarre sa propre collection avec Il giorno della civetta de Leonardo Sciascia. Suivront l'Unità en septembre ("I giorni di storia») et il Giornale ( La biblioteca storica»).

Cette frénésie de livres allegati va se poursuivre et s'amplifier en 2003, lorsque pratiquement tous les quotidiens, mêmes locaux, et les hebdomadaires publieront leur collection.

Il faut souligner que les romans ne sont pas le seul genre de livres allegati. En février 2003 La Repubblica lance sa collection «I grandi del fumetto", et il le fait d'ailleurs en même temps qu'une deuxième série de livres "I grandissimi del '900". Quelques mois après, ce sera le tour des livres d'art : Il Corriere della Sera commence la série des "Classici dell'Arte»; au même moment La Repubblica entame sa collection «l'Enciclopedia La Repubblica", qui est réalisée en collaboration avec UTET. Aussitôt après le Corriere poursuit avec «La storia d'Italia d'Indro Montanelli» et en novembre et décembre avec une série d'encyclopédies, "La biblioteca del sapere». Ce sera ensuite au tour de La Repubblica de revenir au roman, mais cette fois ce sera avec "I classici dell'Ottocento".

On le voit, c'est une course-poursuite. L'énumération de toutes les collections qui ont vu le jour depuis 2002 serait aussi longue que fastidieuse et ne ferait que confirmer l'importance de ce phénomène ${ }^{2}$.

2. Au moins deux "tesi di laurea» ont été consacrées au phénomène du livre allegato : Andrea Cortese et Giacomo Marabese, L'invasione delle edicole: come un'iniziativa editoriale è diventata un fenomeno nazionale, Corso di Laurea in Scienze dell'Educazione, Université de Padoue, Prof. Raffaele Nigro; et Cristian Vaccari, Il libro e il quotidiano: confronto tra le promozioni editoriali "I libri dell'Unità" (I992-1996) e "La biblioteca di Repubblica» (2002). 
Le lancement de la première collection de La Repubblica a été un événement culturel et économique. Pour un coup d'essai, c'était un coup de maître : le chiffre d'affaires de La Repubblica a augmenté en 2002 de $59,64 \%$ par rapport à l'année précédente, alors que l'exercice 200 I avait enregistré une baisse de $0,21 \%$ par rapport à $2000^{3}$. Les autres quotidiens aussi enregistraient des résultats à la hausse, encore que dans de moindres proportions.

S'agissait-il d'un phénomène nouveau? Pas tout à fait. Pour deux raisons.

Lorsque La Repubblica lançait son initiative éditoriale en 2002, ce n'était pas la première fois qu'un journal proposait un livre. En effet, dans les années 1990 l'Unità, sous la direction de Walter Veltroni ${ }^{4}$, avait proposé, parmi les produits dérivés, des cassettes vidéo avec des films rares et aussi des livres dans le but de constituer une véritable «bibliothèque populaire». Et occasionnellement d'autres quotidiens et hebdomadaires, surtout l'été, avaient également proposé des livres, surtout des policiers.

Ce n'était pas non plus la première fois que le livre utilisait ce canal de distribution, à savoir le kiosque. Il faut en effet rappeler qu'en 1965 lorsque l'éditeur Mondadori avait lancé sa collection de poche «Oscar Mondadori» il avait choisi de passer par une publication hebdomadaire diffusée par les dépositaires de presse et, accessoirement, par les librairies. Les kiosques écoulaient en effet $63 \%$ des exemplaires vendus. Là encore ce n'était pas la première tentative de livre à prix réduit, l'antériorité revenait à la BUR; par contre la présentation, le prix (350 lires, légèrement au-dessus du prix d'un hebdomadaire), le choix des titres, les stratégies de marketing, tous ces éléments étaient nouveaux. En 1965 le choix des kiosques permettait de vendre 8400 ooo livres, avec des tirages entre 80 ooo et 100 ooo exemplaires par titre, à une époque où, selon l'Istat, 28,9\% (sans doute plus) des familles italiennes ne lisaient jamais ni livre, ni journal, ni magazine. D'ailleurs, à la fin de cette même année 1965, Mondadori proposait un Oscar mensuel, pour introduire des textes de poésie et de théâtre.

3. Source FIEG.

4. W. Veltroni s'est exprimé de façon très critique sur ces initiatives éditoriales récentes. «L'ex direttore dell'Unità non è affatto entusiasta della piega che il fenomeno ha preso oggi. "Il nostro era un caso in parte diverso da quello di oggi - dice Veltroni - noi proponevamo ai nostri lettori un'offerta omogenea e coerente, giornalistica e culturale. Insomma il lettore non vedeva soluzione di continuità tra l'impostazione del quotidiano e la selezione di prodotti culturali che veniva proposta, era dunque possibile che il giornale diventasse volano di nuova richiesta di cultura ma anche di nuovi contenuti giornalistici." Oggi invece? "Il gadget ha natura autonoma e spesso puramente di richiamo commerciale, in un'escalation di battaglia anche pubblicitaria che concentra l'attenzione solo su quello. Si arriva ad avere la sgradevole impressione che l'allegato sia il quotidiano, piuttosto che il contrario." Quanto al futuro, "è un fenomeno che non si esaurirà a breve, ed è vero che ha aiutato a salvare i bilanci, ma non ha fatto aumentare i lettori".» (Roberta Carlini, "La stampella della stampa», Il Manifesto, Is décembre 2004.) 
Une autre initiative intéressante montre que dans cette escalade on est arrivé à briser des tabous.

En août 2005 l'éditeur Mondadori, propriétaire de la prestigieuse collection "I Meridiani», a surpris le monde des lecteurs et des bibliophiles en distribuant dans les kiosques, allegati à ses périodiques, 30 titres de cette collection. Le premier numéro a été mis sur le marché au prix de I euro. Il s'agissait des romans de Hemingway'. Cette initiative, nous le disions, a surpris car on mettait en vente au prix de 12,90 euros des livres qui en librairie coûtaient normalement 49 euros ( 55 aujourd'hui) ${ }^{6}$. Le texte est le même, seules quelques petites différences au niveau de la reliure et de la qualité du papier permettent de distinguer la collection bon marché de l'édition normale 7 . D'autres part ces livres, qui donnaient aussi un aperçu de la littérature mondiale, étaient distribués avec des périodiques Mondadori, à savoir Panorama, TV Sorrisi e Canzoni, Donna moderna, Tu, Chi, Grazia, c'est-à-dire des revues qui, pour la plupart, n'avaient pas pour cible un public censé être particulièrement friand de littérature, mais davantage de "gossip» et de frivolités.

Cette initiative avait quelque chose d'iconoclaste, ce qui n'a pas empêché Mondadori de récidiver de deux façons : premièrement en distribuant une deuxième collection de Meridiani appelée "I classici collezione» et qui présente, avec le texte en latin ou grec et la traduction en regard, les chefsd'œuvre de la littérature classique (c'est la collection rouge, par rapport à la collection bleue); deuxièmement en poursuivant aussi la première collection qui au début de 2007 était arrivée à son $77^{\mathrm{e}}$ titre.

Naturellement les libraires pouvaient souffrir de cette initiative. Les volumes de "I Meridiani collezione» ont donc été mis en vente en même temps et au même prix chez les dépositaires de presse et chez les libraires. Aujourd'hui encore ils sont disponibles en librairie, dans une édition légèrement différente (première et dernière page), moins liée aux exigences d'une promotion en kiosque; le seul problème c'est que de nombreux titres sont épuisés.

5. Il faut remarquer que déjà en 1965 le premier numéro des "Oscar Mondadori» avait été un roman de E. Hemingway (Addio alle armi).

6. Cette collection, plus que les précédentes, a des implications sociologiques dans la mesure où elle modifie la rapport entre le livre et le lecteur dans son appartenance à un groupe social/économique.

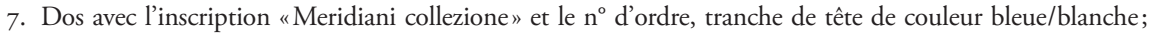
reliure cousue en fil/collée; signet en soie/en tissus synthétique. 
Les Italiens aurait-ils été saisis par une furieuse et irrépressible envie de lire?

Les raisons du succès des livres allegati ne tiennent pas qu'au désir de lire. Il faut dire que toutes ces collections présentaient de nombreux avantages :

I. Le prix était abordable et inférieur à celui des livres vendus en librairie;

2. La qualité typographique et la présentation étaient tout à fait convenables ${ }^{8}$;

3. Les titres proposaient effectivement les grands romans $\mathrm{du} \mathrm{Xx}^{\mathrm{e}}$ siècle, puis du XIX ${ }^{\mathrm{e}}$ siècle, italiens et étrangers;

4. La promotion était assurée par les quotidiens eux-mêmes qui consacraient plusieurs pages à la sortie d'un titre ou par les autres titres du groupe (par exemple L'espresso pour La Repubblica);

5. Ces livres étaient toujours présentés sous forme de collections, ce qui en faisait un "produit d'ameublement", agréable à regarder et pouvant être placé dans un meuble bibliothèque, que le roman soit effectivement lu ou pas;

6. La distribution était faite par des dépositaires de presse. Les avantages de ce système sont doubles : d'une part, il s'agit d'un réseau extrêmement dense et qui offre l'avantage de la proximité (3800o kiosques contre 5000 autres points de vente entre librairies, papeteries et supermarchés); d'autre part, le lecteur occasionnel, qui peut être intimidé par le professionnalisme du libraire, se sent plus à l'aise chez son dépositaire de presse chez qui il se rend habituellement pour acheter son journal;

7. Enfin, pour ce même lecteur occasionnel, le fait qu'un titre soit proposé par le journal qu'il achète habituellement et qui lui «recommande» ce livre, est un gage de qualité littéraire, une sorte de label dont il peut profiter.

Ces initiatives ont été critiquées en partie et pour diverses raisons. Elles présentent en effet des aspects négatifs ou contestables.

I. La dévalorisation du livre. La valeur intrinsèque du livre, donc de l'œuvre, est affectée par ces opérations qui donnent l'impression que

8. La publicité pour la première collection de La Repubblica faisait valoir la qualité éditoriale de ces livres : "Rispetto ad altre serie di libri stampati per essere venduti con i quotidiani, questa collana ha caratteristiche straordinarie, prima di tutto, la qualità editoriale. Si tratta, infatti, di veri prodotti da libreria: carta di pregio, grafica elegante, copertina rigida e sovracopertina." 
le livre est une marchandise comme une autre et qu'en réalité le prix affiché en librairie est largement surévalué;

2. La fétichisation de l'objet-livre. On insiste, dans toutes les campagnes promotionnelles, sur l'aspect «série» et on donne au lecteur la possibilité de compléter la collection : le titre perd là aussi sa valeur propre et devient un numéro de la série et un objet d'ameublement;

3. L'infantilisation du lecteur. La liberté de choix est menacée par la "collection» : une fois que l'on a commencé, on continue par fidélité ou par habitude. De surcroît le lecteur n'a plus à se poser la question car on a déjà choisi pour lui ce qu'il doit lire;

4. Le conditionnement publicitaire. Tous les journaux qui proposent des livres allegati assurent aussi une intense campagne promotionnelle : encadrés dans le journal, annonces et publicité, interviews et pages culturelles sur l'auteur. Naturellement ces campagnes font appel à des personnalités du monde littéraire et non littéraire qui écrivent habituellement dans le journal. Avec quelques absurdités'. L'impression de matraquage publicitaire est parfois très nette;

5. En 2005 Mario Lavagetto a émis des réserves sur l'intérêt de ces "bibliothèques" de "chefs-d'œuvre» car d'une part ces collections peuvent gêner des tentatives plus risquées et plus novatrices d'autres éditeurs, d'autre part cette "invasion" est concomitante à une certaine occultation de la critique littéraire. Le résultat est que le texte est pris tel quel et abordé sans aucune "médiation exégétique». Il est vrai aussi que depuis deux ans les éditeurs d'allegati se sont rattrapés en proposant des histoires de la littérature : par exemple la Letteratura italiana d'Emilio Cecchi et Natalino Sapegno, avec le Corriere della Sera en 2006, ou encore la Letteratura italiana dirigée par Alberto Asor Rosa chez L'espresso.

\section{Littérature et médias}

Ce phénomène serait à la rigueur éditorial et non littéraire s'il se limitait à la diffusion d'œuvres "classiques", qui ont déjà fait leurs preuves et

9. On a vu par exemple Adriano Sofri chargé de la présentation du volume sur Calvino dans la collection des Meridiani. Sofri a dû avouer qu'il n'était pas la personne la mieux placée pour présenter Calvino. «Ho accettato di presentare l'uscita, con Panorama, del primo volume dei Meridiani dedicati ai romanzi e racconti di Italo Calvino, e subito ho cominciato a preoccuparmi, perché amo Calvino e trovo bellissimi i Meridiani, ma non sono abbastanza preparato. Allora ho chiesto a Carlo Ginzburg, che di Calvino fu amico e interlocutore, di parlarne con me e con voi. Cominciando dal suo primo incontro con Calvino." (Panorama, 5 septembre 2005.) 
sont reconnues comme littéraires. Mais une collection récente a changé la donne. En effet, à l'initiative du Corriere della Sera et de l'éditeur RCS, au cours du printemps 2007, une série de 15 récits inédits à été publiée sous l'appellation imagée "Corti di carta» au rythme de deux récits par semaine. Vendus au prix de I euro, d'un format strictement identique, ce sont de

brefs romans de poche qui, grâce à leur présentation soignée, à leur extrême maniabilité (64 pages), à la variété des genres et des styles proposés, offrent un aperçu ample et riche de la littérature contemporaine. Une collection unique, idéale aussi bien pour tous ceux qui ont l'habitude de lire et qui veulent découvrir les écrivains italiens les plus connus aux prises avec de nouvelles aventures, que pour ceux qui ont peu de temps libre à consacrer à la lecture et qui préferent des histoires éclair.

Les auteurs qui ont collaboré à cette série sont Roberto Saviano, Susanna Tamaro, Giorgio Faletti, Dario Fo, Alessandro Piperno, Fabio Volo, Melania Mazzucco, Sandro Veronesi, Federico Moccia, Walter Veltroni, Dacia Maraini, Gianantonio Stella, Isabella Bossi Fedrigotti, Valerio Massimo Manfredi et Alberto Bevilacqua.

Actuellement nous ne savons pas si cette initiative a été couronnée de succès ni quelles sont les retombées économiques ou d'image pour l'éditeur et les auteurs. On ne sait pas non plus si cette formule sera reprise avec d'autres écrivains ou si les textes seront proposés à nouveau sous forme de recueil.

En tout cas cette initiative est intéressante à plus d'un titre. Car force est de constater qu'elle trouble le jeu des rapports entre l'auteur et son œuvre, entre l'auteur et son lecteur. Car d'une part, l'auteur écrit sur commande, avec des contraintes précises de temps et de format, d'autre part, il atteint le lecteur par un circuit qui n'est pas le circuit traditionnel des librairies, mais par celui des dépositaires de presse et dans les conditions particulières qui étaient celles, autrefois, des "paralittératures ${ }^{\mathrm{IO}}$ ".

Io. Pour une discussion sur les limites, l'«incertitude», les frontières du mot et de la notion, voir Daniel Couégnas, Introduction, p. II-24 et Alain-Michel Boyer, La Paralittérature, p. 13-22. La diffusion par les dépositaires de presse et le prix sont les deux caractéristiques principales qui justifient cette confrontation. "Les paralittératures sont nées vers le milieu du $\mathrm{XIX}^{\mathrm{e}}$ siècle de la conjonction de plusieurs phénomènes : la reconnaissance du roman comme une forme littéraire à part entière, le développement des moyens d’impression industrielle, la nouvelle diffusion de l'écrit grâce à la multiplication des voies de chemin de fer et des routes. Le succès du "livre bon marché" passe par les kiosques de gare implantés par Hachette [...].» (Fondanèche, Paralittératures, p. 9.) Rappelons par ailleurs que l'expression «littérature de gare» a un rapport étroit avec la concession de kiosques en gare obtenue en 1852 par Louis Hachette qui créa, entre autres, "La bibliothèque des chemins de fer». La Librairie Hachette signera en 1882 un contrat d'exclusivité pour les librairies installées dans les gares. 
En effet, plusieurs éléments rappellent les modes de production et de diffusion de la littérature "populaire» au XIX ${ }^{\mathrm{e}}$ siècle et de certains types de production réunis sous l'étiquette "paralittératures ${ }^{\mathrm{II}}$ ".

Des éléments externes ou sociaux et des éléments internes ou textuels ${ }^{12}$ peuvent permettre d'ébaucher dialectiquement une réflexion sur "Corti di carta».

Examinons quelques critères externes de cette collection.

I. Le statut de l'écrivain qui écrit pour cette collection est reconnu : il s'agit d'auteurs légitimés par l'institution littéraire même si, pour certains, on ne peut pas parler véritablement d' «auteur littéraire».

2. La liberté-gratuité de l'acte d'écrire : la plupart des écrivains de la série "Corti di carta» se refusent à dire qu'il travaillent sur commande ${ }^{\mathrm{r}}$. Les interviews par lesquelles RCS assure la promotion de sa collection font état d'une assez grande unanimité pour réfuter l'idée que les auteurs travaillent sur commande. Ainsi Susanna Tamaro :

Je n'ai jamais écrit sur commande, sauf peut-être deux ou trois fois, au début de ma carrière, lorsque je ne connaissais pas encore ce métier. Depuis je ne l'ai plus fait, puisque je me suis rendu compte que je ne suis pas capable de travailler en suivant des directives édictées par d'autres. J'ai une créativité rebelle, qui ne m’obéit pas [...].» (Corriere della Sera Magazine, I2 avril 2007.)

Elle affirme aussi ne signer de contrat qu'après avoir achevé son travail et en être "convaincue». Quant à Dario Fo, il botte en touche. Pour lui, c'est un faux problème :

Le problème n'est pas savoir s'il est plus gratifiant ou plus restrictif de travailler sur commande ou en toute liberté, à savoir s'il vaut mieux suivre les directives d'autrui ou ne suivre que ses propres désirs, son rythme, ses aspirations. Le problème est un autre : il faut que le travail sur commande coïncide avec les intérêts de celui à qui on l'a demandé, avec sa façon de voir. (Magazine, I9 avril 2007.)

Quelques écrivains admettent qu'ils ont écrit sur commande. Alessandro Piperno reconnaît qu'il trouve "salutaires" des échéances et des contraintes, car les règles l'aident à se discipliner. Dacia Maraini et Gianni Veltroni aussi assument cette contrainte.

II. Ce terme controversé ne sera pas utilisé ici comme un jugement esthétique, mais comme un critère opératoire qui permet d'opposer deux types de production littéraire pour tenter d'en caractériser les aspects les plus significatifs.

I2. La terminologie et les critères internes sont empruntés à Alain-Michel Boyer, Frontières du littéraire, p. 57.

13. Certains de ces écrivains sont des collaborateurs habituels du Corriere della Sera ou des publications du même groupe (Stella, Bossi Fedrigotti). 
La commande est une incitation - affirme Dacia Maraini - et il n'y rien de mal à écrire selon des indications données par d'autres. C'est ainsi que sont nées, par exemples, toutes mes pièces : à la demande d'une troupe ou d'un acteur [...]. Au fond les fantasmes qui m'habitent peuvent entrer dans une «maison» ou dans une autre. (Magazine, 24 mai 2007.)

Cette réticence à admettre qu'ils ont écrit sur commande montre que pour la plupart de ces écrivains le problème est réel et qu'ils en ressentent une gêne, comme si cela modifiait le statut du livre écrit dans ces conditions ou de l'auteur soumis à ces contraintes.

3. Le circuit de distribution : "Corti di carta» arrive au lecteur par les dépositaires de presse. On peut sans doute opposer le circuit des librairies et celui des kiosques comme étant caractéristiques, surtout à des époques plus lointaines, de deux types de production littéraire.

4. La périodicité est souvent une des caractéristique des collections "paralittéraires", tout comme le prix qui en fait un produit très abordable. Les "Corti di carta» se conforment à ces critères.

Qu'en est-il des critères internes ou textuels?

I. Il n'y a pas d'idée de série, dans la mesure où les "Corti di carta» n'offrent pas «à chaque fois, une nouvelle version d'un schéma canonique qui transcende l'ensemble des textes" (Boyer, Frontières, p. 57-58). Il n'y a pas d'idée de série, de personnages-types présents en général dans les ouvrages de paralittérature; il s'agit de sujets très différents, de cadres différents, de styles différents.

2. Il n'y a pas de dominante, c'est-à-dire d'un «moteur de la fable ou cellule d'organisation qui assure le rôle de cohérence narrative» (ibid., p. 58); une grande diversité de motifs et de ressorts de l'action traversent ces récits hétérogènes.

3. Il n'y a pas de décor qui renvoie à une doxa, comme dans la série "qui n'a souvent pour référent que l'ensemble des récits qui la composent» (ibid.).

4. Le contrat de lecture ne correspond pas à celui de la série : dans la série, le programme narratif est prévu d'avance, le lecteur s'attend à un final en conformité avec la loi du genre.

Les critères internes ne semblent donc pas apparenter les "Corti di carta" aux publications réunies sous l'étiquette "paralittératures" alors que plusieurs critères externes suggèrent ce rapprochement. On peut donc parler d'une forme hybride : littéraire pour les caractéristiques textuelles 
et pour le statut de l'auteur, "paralittéraire» pour les caractéristiques externes : le circuit de distribution, l'écriture sur commande, la périodicité (prix, sortie périodique et aspect matériel de collection).

En somme, la série "Corti di carta» semble brouiller les pistes, se démarquer des initiatives précédentes, modifier le rapport entre l'auteur et son œuvre, donner une autre image de la littérature, instaurer une autre relation entre l'auteur et le lecteur.

\section{Bilan et perspectives}

Les éditeurs italiens de libri allegati sont pris dans une spirale : le produit tient encore, mais pendant combien de temps? Le rapport annuel de l'AIE "Libri e contenuti editoriali: lo stato dell'editoria in Italia nel 2006" parle de saturation du marché. Toutefois, si 2006 a connu un recul du chiffre d'affaires des allegati ( $-9 \%$ en 2006 , mais $+43 \%$ en 2004 et $+\mathrm{II}, 9 \%$ en 2005 ) il a réalisé tout de même un volume de 489 millions d'euros. Faut-il interpréter cet arrêt de la progression comme un signe d'essoufflement?

Ce n'est pas la première fois que la littérature emprunte de nouveaux médias, en particulier le circuit des dépositaires de presse : il suffit de penser au roman feuilleton du XIX ${ }^{\mathrm{e}}$ siècle, ou encore à des formes de présence de la littérature, et de la culture en général, dans la presse : l' elzeviro" et la "terza pagina». Mais cette forme d'édition littéraire peut nous dire quelque chose de la mentalité et de la pratique culturelle des Italiens et des Français. L'attitude spregiudicata des Italiens montre à la fois l'esprit d'initiative, l'attitude pragmatique dans le domaine culturel et l'absence de contrainte extérieure que l'on observe aussi dans d'autres secteurs de la société italienne $\mathrm{I}^{\mathrm{I}}$.

En France dans les kiosques se sont imposés les produits dérivés, mais pas les œuvres «littéraires». Une tentative du Point a été aussitôt contrée par les libraires. Cet hebdomadaire, qui en 2003 avait essayé de rééditer l'exploit de La Repubblica en distribuant 70000 exemplaire des Contes de la Bécasse de Maupassant, a été l'objet d'une action judiciaire intentée par le Syndicat national du livre et les libraires. L'hebdomadaire a dû suspendre ses publications après avoir commencé sa collection "Les classiques du Point» avec Maupassant, Proust (Un Amour de Swann, n ${ }^{\circ} 585$ ), Balzac (Les Chouans, $\mathrm{n}^{\circ}$ 1587) et Sun Tse (L'art de la guerre, $\mathrm{n}^{\circ}$ 1588).

I4. Il faudrait citer aussi, parmi les initiatives novatrices, qui ont été ensuite copiées à l'étranger, les livres Millelire de "Stampa alternativa», lancés en 1989 avec la Lettera sulla felicità d'Épicure. 
En janvier 2007, Serge Eyrolles, président du Syndicat national du livre, auditionné par la commission des Affaires culturelles du Sénat sur l'avenir du secteur de l'édition, réaffirmait son opposition à la vente de livres avec les journaux :

Le SNE est hostile aux opérations consistant à coupler ventes de presse et de livres, mêmes si elles permettent d'élargir le marché, car elles entraînent un détournement de la clientèle au détriment des libraires [...] et parce que la faiblesse des prix ne donne pas une juste image du livre.

La distribution de produits dérivés par les journaux a toutefois débuté en janvier 2004 lorsque Le Figaro a proposé une collection de DVD au prix de 3 euros, vendus avec son édition du week-end. Le Monde ne pouvait être en reste et lançait sa propre collection de DVD (en augmentant aussi les ventes du journal de $15-20 \%$ ) et récidivait en 2005 avec une série de livres d'art.

Aujourd'hui plusieurs initiatives éditoriales sont en cours (citons seulement la série de $\mathrm{BD}$ de Blake et Mortimer chez Le Monde et le dictionnaire Littré pour Le Figaro), d'autres sont annoncées, mais on ne sait pas encore ni quand ni par qui sera franchie la ligne blanche qui sépare la distribution de la presse de la distribution des livres.

Ce qui se passe en Italie sera sans doute repris en France. La forte diffusion, ces derniers années, d'auteurs très "populaires » qui n'écrivent pas dans des "séries" de couleur (rose, noire, jaune...) mais sont distribués par les circuits officiels pourrait préluder à des initiatives comme celle de "Corti di carta». Mêmes si les mentalités, la culture, les comportements ne sont pas tout à fait les mêmes, d'un côté et de l'autre des Alpes, les contraintes économiques finiront par s'imposer et la littérature réempruntera ces «nouveaux» médias.

\section{Bibliographie}

Boyer Alain-Michel, Frontières du littéraire, disponible sur <http://www. lettres.univ-nantes.fr/editions/Boyer/Boyer.html>.

—, La Paralittérature, Paris, PUF, collection "Que sais-je?», I992.

CouÉgnas Daniel, Introduction à la paralittérature, Paris, Le Seuil, collection «Poétique», I995.

Fondanèche Daniel, Paralittératures, Paris, Vuibert, 2005.

Lavagetto Mario, Eutanasia della critica, Turin, Einaudi, 2005. 
